\title{
Optometría clínica, desde la práctica hacia la teoría
}

\author{
José MARÍA SÁnCHEZ GonZÁLEZ \\ Universidad de Sevilla. Facultad de Farmacia \\ Departamento de Física de la Materia \\ Condensada. Área de Óptica \\ jsanchez80@us.es \\ ORCID: https://orcid.org/0000-0003-0450-7717 \\ D.O.I.: http://dx.doi.org/10.12795/JDU.2018.i01.53 \\ Pp.: 939-956
}

\section{Resumen}

La comunicación se presenta en estas Jornadas Docentes versa sobre la inversión de secuencia de actividades en una clase (flipped Classroom, Flipped Learning o clase invertida) (Barnett \& Society for Research into Higher Education., 2005) de una asignatura de último curso del Grado en Óptica y Optometría y el Doble Grado en Farmacia, Óptica y Optometría.

La experiencia ha resultado muy positiva y muy bien recibida por los alumnos. La participación también ha sido elevada y desde el punto de vista del aprendizaje, la atención y motivación mostrada por los alumnos ha ayudado a la comprensión y entendimiento de los contenidos conceptuales y procedimentales.

Palabras Claves: Consulta de Optometría, Grado en Óptica y Optometría, Innovación Docente y Gamificación 


\section{Contexto de la intervención}

La asignatura de "Consulta de Optometría. Casos Clínicos Especiales", pertenece al cuarto curso del Grado en Óptica y Optometría y al sexto curso del Doble Grado en Farmacia, Óptica y Optometría. Tiene 75 alumnos, que constituyen un único grupo de teoría, 3 grupos de seminarios y 7 grupos prácticos de 11 alumnos (impartidos por cuatro profesores). La teoría son 15 clases de 1 hora, los seminarios dos sesiones de 2.5 horas. Las prácticas están organizadas en 11 sesiones de 3.5 horas cada una y se evalúan mediante un examen final práctico. Por lo que el CMD serán 3 CMD encadenados formando uno de asignatura completa.

El aula dónde se imparte la docencia es un aula de la parte antigua de la facultad de Farmacia (capacidad para 100 alumnos), tantos los seminarios como las prácticas son en los gabinetes de optometría con los instrumentos y maquinarias necesarios para realizar un examen visual a pacientes. A continuación, se muestra una imagen de uno de los gabinetes para que se pueda apreciar el tamaño y la distribución de este (Figura 1).

La parte práctica de la asignatura tiene un peso del $60 \%$ y el contenido teórico un $40 \%$; los alumnos tienen que realizar dicha actividad con pacientes reales y realizar exámenes visuales utilizando los procedimientos explicados en clases de teoría.

Jornadas de Formación e Innovación Docente del Profesorado I № 1 (2018) Esta obra se distribuye con la licencia Creative Commons 


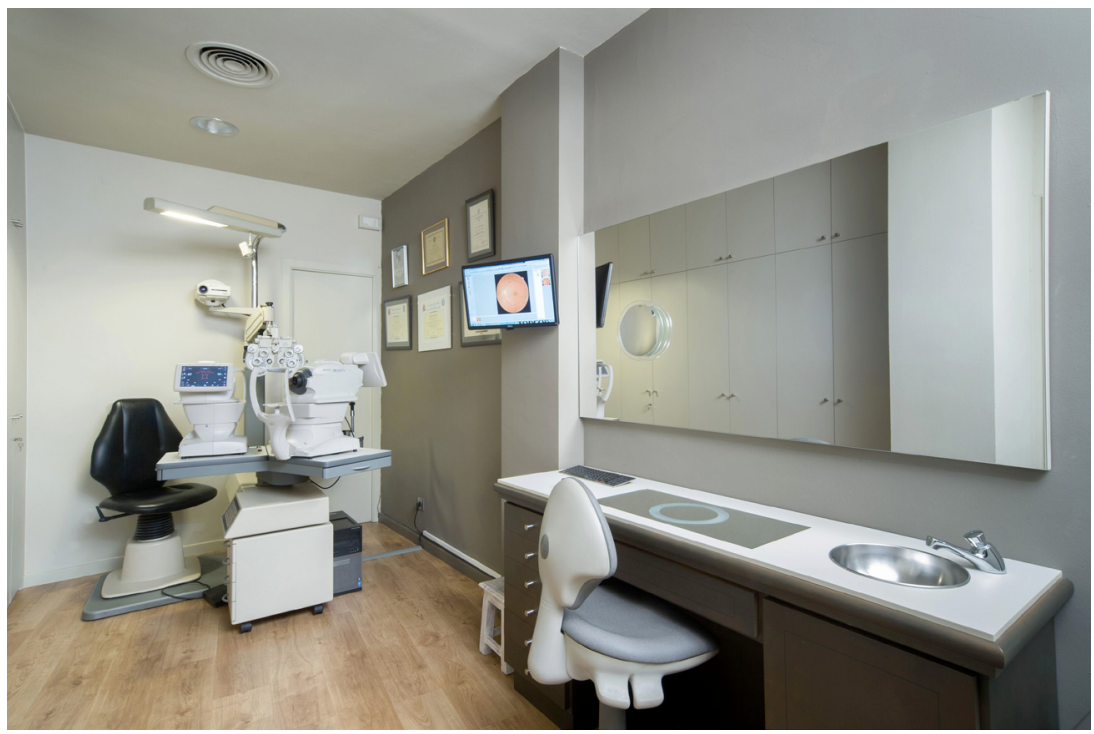

Figura 1. Distribución de un gabinete de optometría en la Facultad de Farmacia

\section{Diseño previo del ciclo de mejora docente}

Mi modelo ideal sería uno basado en la reelaboración de idea de los estudiantes. Primero el planteamiento de un problema o un caso (Aprendizaje basado en resolución de casos mediante el método de Flipped Learning) (Ashwin, 2015). Concretamente se trata de un caso de optometría y problemas de la visión. El alumno deberá apoyarse en los conocimientos anteriores de Optometría I y Optometría II para poder resolver estos casos. En un segundo lugar tendríamos la elaboración de las ideas de los estudiantes para resolver el caso. En un tercer punto, puesta en prácticas de las ideas de los estudiantes. Por último, establecer unas tesis que engloben lo expuesto por los alumnos con las correcciones oportunas del docente.

Dado que la parte teórica de la asignatura es una hora por semana durante las 15 semanas de cuatrimestre, el modelo real sería un modelo basado en la práctica y extracción de la teoría a partir de los casos. Por lo que el caso práctico sería la base de la clase, lo que supone una aplicación al mundo real de la Optometría. 
Respecto al mapa de contenidos, se presenta el mapa de contenidos de los dos primeros CMD que corresponden con teoría y seminarios. El CMD específico de prácticas se presentó como comunicación en las Jornadas Docentes de 2017 y se aplicará igual. Se representa el curso natural de un caso clínico de optometría. Desde la identificación inicial y reconocimiento de la patología hasta la aplicación del tratamiento, pasando por la observación de las pruebas del caso, la planificación de las pruebas a seguir y la derivación al oftalmólogo si fuera necesario.

De forma complementaria a estos contenidos conceptuales y procedimentales, los alumnos también desarrollan contenidos actitudinales, en muchas casos son los más valiosos y ni si quiera se dan cuenta que los están adquiriendo. Entre ellos están; el alumno toma consciencia del caso (Musthafa \& Sajila, 2014), se interesa por el tratamiento y rehabilitación del pacientes, interioriza los conocimientos (Mizoguchi, Dillenbourg, \& Zhu, 2006), se esfuerza por conseguir un resultado satisfactorio ya que se trata de un caso real. Y por último valora y es sensible al proceso de resolución del caso (Jackson \& Simpson, 1994). El CMD 2 consistió un repaso de la Teoría de Optometría I y II para preparar a los alumnos para las prácticas. 
Mapa de Contenidos CMD 1 - Asignatura Completa

José María Sánchez - Consulta de Optometría

Grado Óptica Optometría - REFID - Curso 2018/2019
Azul - Conceptual

Rojo - Procedimental

Verde - Actitudinal

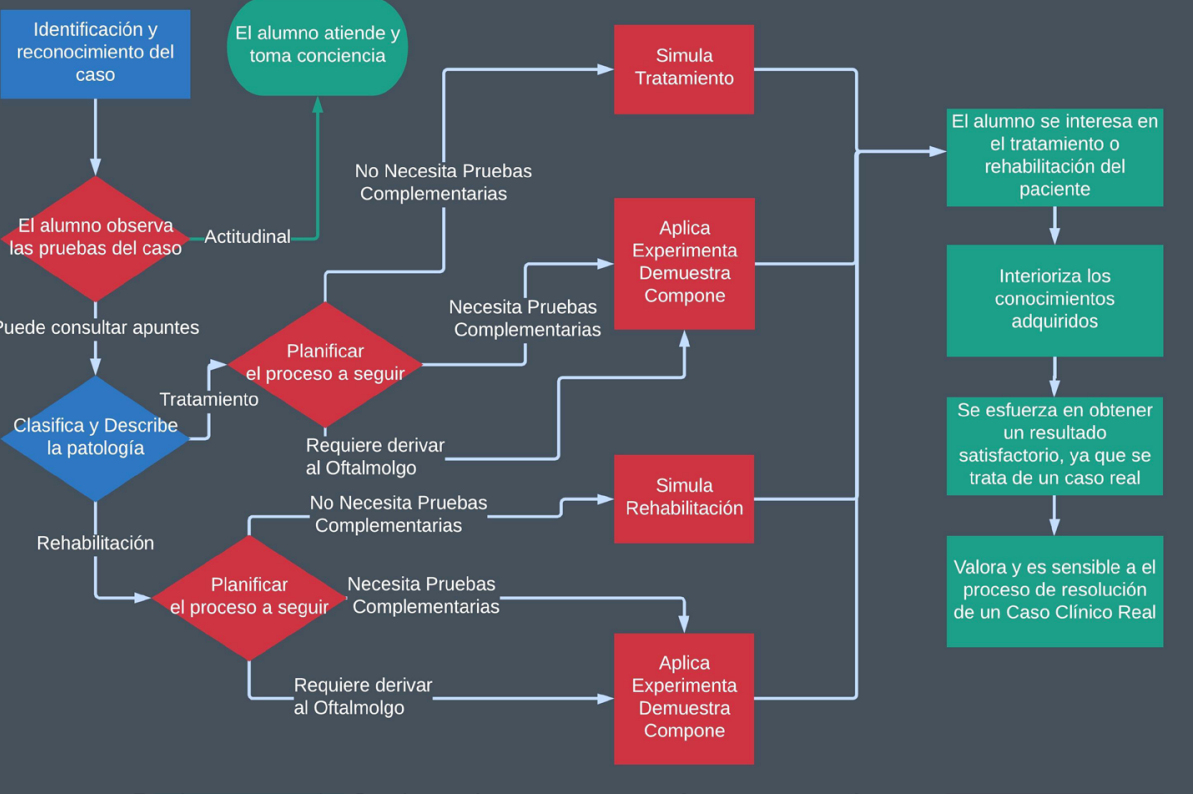

Figura 3. Mapa de contenidos

Respecto a la secuencia de actividades, a continuación, se detallan todos los pasos que se realizaron dentro de cada una de las sesiones de teoría. Respecto al CMD 2, las actividades fueron el repaso de los diferentes temas explicados durante Optometría I y Optometría II. En este caso al ser dos sesiones de 2.5 horas, se repartieron durante la primera sesión los contenidos básicos de Optometría I y durante la sesión dos los contenidos de la asignatura de Optometría II, que son algo más avanzados. La secuencia de actividades del CMD 3 fue idéntica a la que se presentó en la Jornadas Docentes Universitarias de 2017.

Es importante destacar de la secuencia de actividades que el bloque de los casos del 1 al 5 y del 6 al 10 (en color verde en la figura) se repitieron por cinco veces cada uno para completar los 10 casos clínicos. 
El primer día de clase se estableció en el ciclo de mejora para la presentación de la asignatura y la entrega de cuestionarios. Se expuso el programa de la asignatura, se repasaron los criterios de evaluación que se incluían dentro del programa docente donde se explicaba el porcentaje de la teoría y la práctica. Una vez concluido entregué un cuestionario de conocimientos previos donde los alumnos tenían que auto valorar sus conocimientos antes de iniciarse en la asignatura

\section{Aplicación del CMD}

El primer día de clase, se presentó para todos los alumnos un cuestionario inicial de conocimientos previos (Elton, 2001). En el cuestionarios se preguntaron tres cosas;

1. ¿Soy capaz de realizar un examen optométrico completo, incluyendo estudio de la visión binocular, acomodación, refracción objetiva y subjetiva y pruebas complementarias (Amsler, retinografía, oftalmoscopia, Hess Lancaster)?

2. Tengo los conocimientos suficientes para diagnosticar a los pacientes de problemas optométricos.

3. Tengo los conocimientos suficientes para elaborar un tratamiento optométrico desde el punto de vista refractivo o de terapia visual.

Los resultados obtenidos en esta encuesta de conocimientos previos fue la siguiente; en las tres preguntas se valoró desde; necesito más conocimientos hasta tengo los conocimientos suficientes para evaluar un caso clínico.

La escala fue desde 1 a 5 puntos y los resultados obtenidos fueron: Respuesta $1-8,8 \%$, Respuesta $2-28,1 \%$, Respuesta $3-43,9 \%$, Respuesta 4 - 19,3\% y Respuesta $5-0,0 \%$. En la pregunta 2 se valoró si los alumnos consideraban que tenían los conocimientos suficientes para 
diagnosticar los problemas optométricos de los pacientes de los casos clínicos. En este caso los resultados obtenidos fueron: Respuesta 1-8,8 \%, Respuesta $2-21,1 \%$, Respuesta $3-50,9 \%$, Respuesta $4-17,5 \%$ y Respuesta $5-1,8$ $\%$.

Finalmente, la tercera pregunta evaluaba la capacidad de los alumnos para aplicar el tratamiento optométrico desde un punto de vista refractivo o bien según terapia visual. En este caso los resultados obtenidos fueron los siguientes: Respuesta 1-22,8 \%, Respuesta 2 - 43,9\%, Respuesta $3-31,6 \%$, Respuesta $4-1,8 \%$ y Respuesta $5-0,0$ $\%$. Estas cuestiones quedan recogidas en las escaleras de aprendizaje previas representadas en la Figura 5.

Por lo tanto, podemos concluir como los alumnos consideran que tienen los conocimientos suficientes para realizar un examen optométrico e incluso para realizar un diagnóstico optométrico pero que no son capaces de elaborar un tratamiento refractivo o relacionado con terapia visual.

Esto me ayudó para abordar la asignatura desde un punto de vista más práctico (Paroutis, Angwin, \& Heracleous, n.d.) y relacionado con la resolución de problemas basados en casos prácticos para que los alumnos fuesen capaces de aplicar los contenidos teóricos y procedimentales a situaciones reales en forma de casos.

A modo de resumen en cuanto la asistencia para la clase de media suele ser entre 62-67 alumnos de los 75 matriculados. Considero que tengo un grado de asistencia muy alto del $86,6 \%$. La asistencia está descrita en el programa docente como no obligatoria pero sí valorable positivamente. Ya que el primer día se explicaron los criterios de evaluación de la asignatura y los criterios de corrección del examen todos los alumnos estuvieron muy atentos. Entre los 75 alumnos, tres me comentaron por correo 
electrónico que nunca asistirían a clase porque actualmente se encuentran trabajando o con una situación personal complicada. Les expliqué que las prácticas son lo único obligatorio y qué las clases se irían subiendo a la plataforma.

En la primera clase en la que se abordaba el primer caso clínico, quise explicarse como iba a sucederse el transcurso del cuatrimestre. Se abordarían 5 casos clínicos orientados a gabinete de optometría, esos casos clínicos están inspirados en pacientes reales que vimos en prácticas el curso anterior. Después, se proporcionarían los contenidos teóricos correspondientes a esos casos clínicos. Se continuaría con un bloque de otros 5 casos clínicos orientados a la práctica clínica de optometría, en este caso, serían casos obtenidos de mi práctica profesional en la clínica donde trabajo por las tardes. Luego habría otra sesión de contenidos teóricos relacionada con esos 5 casos clínicos. Y finalmente se cerraría con una sesión de dudas, resumen de casos y juego final donde se valoraría la puntuación obtenida en el juego КАНOOT(Alderman, 2014).

En cuanto al desarrollo de las sesiones, los primeros 5 minutos los utilizo para cargar la presentación en el ordenador, preparar mis cosas, firmar la asistencia y en ocasiones algún alumno se acerca a mi mesa y me pregunta alguna duda o problema que tiene con los grupos de prácticas. Para ajustarme al tiempo marcado, le digo que lo tengo que valorar tranquilamente y que me envie un correo electrónico. Considero que 60 minutos de clase es muy poco y no resultaría eficiente resolver dudas individuales y por eso les remito a mi correo electrónico.

A continuación, son necesarios entre 2-3 minutos para que se callen todos, siempre esta parte es muy pesada. Algunos alumnos ya se encuentran en silencio y miran a los compañeros esperando que paren de hablar y se empiece la clase. En estos momentos, yo me quedo en silencio y se 
suelen callar entre ellos. Pero esos 2-3 minutos de rigor siempre suceden.

Empiezo la presentación del caso, explico la anamnesis del paciente, y voy dando pinceladas de posibles patologías o de posibles pruebas y voy avanzando en las pruebas realizadas y en los resultados obtenidos. En muchos casos, como presento el trabajo realizado por compañeros alumnos del curso pasado, hago correcciones sobre lo que está escrito y ellos tienen que ir modificando sus apuntes ( $\mathrm{Bi}-$ ggs, 2003).

El primer día, apenas hubo dudas y casi todos los alumnos estuvieron anotando sobre la presentación en Power Point, intenté que la colaboración fuese mayor pero solo se oían débiles voces. Durante la clase, estaba andando continuamente de izquierda a derecha y observaba como su mirada me iba siguiendo por la sala. Realmente como era el primer día, no me atreví a subir entre las gradas de los alumnos (Jaques, 2000). Me hubiera gustado hacerlo. Casi finalizando la clase, uno de los alumnos se quedó muy pensativo y me preguntó si está iba a ser la manera habitual de dar las clases. En ese sentido pensé si me lo preguntaba desde un punto de vista negativo, pero cuando termino de explicarse, entendí que realmente le estaba gustando lo único que al no estar acostumbrado a explicaciones basadas en casos clínicos reales no sabía cómo iba a ser el transcurrir del cuatrimestre.

En los sucesivos días, los casos del 2 al 5 se expusieron, casi todas las clases siguieron el mismo patrón en cuanto al orden de los acontecimientos. Lo que si observé fue que la participación de los alumnos fue aumentando progresivamente (Elton, 2006). La asistencia se mantuvo igual, pero no tenía intención de que subiese. Realmente tengo controlado los que faltan y creo saber los motivos. En total, hay 5 alumnos que no asisten, a pesar de poder hacerlo. El resto tiene motivos especiales. 
La asistencia la cuantifico con la participación en un juego de KAHOOT al final de la clase. Al principio utilizaba solo preguntas con respuesta del tipo; estoy de acuerdo o no estoy acuerdo. Me servía para dividir la clase y establecer un pequeño debate. Observé que los alumnos perdían el interés con este tipo de modo de juego y lo cambié a tipo trivial con 4 opciones y con puntuación. En este caso, son los alumnos que han fallado los que me indican durante la clase porque creen que su respuesta es correcta y la defienden. Durante las primeras clases esto no pasaba, pero ahora al final los alumnos están adquiriendo una confianza que hace que sean capaces de exponer sus justificaciones. Algo que considero muy positivo.

Durante el tiempo que dura la gamificación observo como existe una revolución en la clase y el interés despierta. Intento siempre que sea un periodo de tiempo corto para que no se aburran de estas herramientas de gamificación. Al principio me encontraba inseguro, pero también ha sido beneficioso impartir los curso de "Taller práctico de Gamificación en Aula" a través del ICE, ya que he intercambiado opinión con otros compañeros docentes que también están interesado en el mundo de la gamificación o se están iniciando en él.

A lo largo de los siguientes días, observé como los alumnos tomaban menos apuntes y estaban más pendientes de mi explicación. Además, las preguntas o cuestiones que iban desarrollando eran cada vez más cercanas a preguntas que ellos se estaban planteando de verdad. Por ejemplo: ¿Cómo podríamos solucionar el problema al paciente?, ¿En todos los casos debemos actuar igual?, si nos pasa en consulta, ¿Cómo debemos reaccionar? .... Este tipo de cuestiones era las que yo quería que fueran surgiendo en las clases para que vieran la necesidad del aprendizaje basado en casos y su utilidad en el mundo real que les espera próximamente. 
En la sesión que correspondía con el caso 5 fue la primera en la que se realizó la grabación de las clases por parte de Soraya Hamed Al-Lal (mi coordinadora en el proyecto de docencia universitaria que organiza el grupo de Rafael Porlán y Nicolás de Alba). Tenía miedo de que el comportamiento no fuese el real, pero pasado los primeros 5 minutos los alumnos continuaron haciendo las mismas preguntas que en la última sesión y reaccionaron igual que si no hubiera cámaras. Yo me olvide de que me estaban grabando en algunos momentos.

En la sesión de debate que ocurrió después de la exposición de los 5 casos clínicos, sí que observé que el interés bajo mucho. Hasta el punto de que les comenté si tenían examen o algún evento importante porque no estaban atendiendo como siempre. Creo que, al hacerlo al revés, los alumnos estaban escuchando datos repetidos que ya habíamos abordado en sesiones anteriores y por eso no prestaban atención. En algunos momentos, incluso yo me di cuenta de que estaba repitiendo cosas que había dicho en otras clases y que se estaba haciendo aburrido. Cómo la sesión de teoría se tiene que repetir pasados los casos clínicos del 6 al 10, me estoy planteando cambiar la manera de hacerlos.

Las clases de los casos 6 y 7 fueron con una mecánica similar a las de los otros 5. Los alumnos parecían algo más perdidos porque el contenido de los casos era más complicado y además eran cosas con las que todavía no habían trabajado, ni visto en prácticas por lo que preguntaron menos y se dedicaron a tomar más apuntes.

El relato termina en esta sesión, la fecha límite de entrega de la comunicación se estableció el 19 de noviembre y el cuatrimestre aún no ha finalizado. Por lo que quedan sesiones por dar y el examen final también tiene que hacerse. En cuanto al resto de sesiones en las que se abordan casos clínicos la secuencia de actividades va a ser la misma que en clases anteriores y realmente no espero 
ningún cambio en la actitud de los alumnos, respecto de la que llevamos hasta ahora.

Para la evaluación del ciclo de mejora docente se utilizaron las escaleras de aprendizaje. En este sentido se volvió a pasar el cuestionario de conocimientos previos, pero ahora para evaluar conocimientos finales. Los alumnos tuvieron que contestar de nuevo la misma pregunta una vez transcurrido todo el ciclo de mejora docente de asignatura completa y fueron ellos mismos lo que se autoevaluaron respecto al aprendizaje obtenido durante estos meses. Por lo tanto, el método de evaluación fue autoevaluación a través de escaleras de aprendizaje.

Los resultados obtenidos están descritos en la Figura 5. Brevemente, podemos llegar a conclusión que en la pregunta 1, en la que se evalúa sobre los conocimientos teóricos para realizar un examen optométrico por los alumnos, no ha existido apenas mejora del aprendizaje, algo que era de esperar. Esto es debido a que la asignatura es del último curso de Grado de óptica y Optometría y último curso del Doble Grado de Farmacia, Óptica y Optometría y no estaba destinada para aumentar conocimiento desde el punto de vista teórico sino más bien enfocado a la práctica. Esto ha supuesto la clave diferenciadora respecto a la asignatura en cursos anteriores. Es el primer año en el que soy coordinador de la asignatura y además se enfoca desde la práctica. En cursos anteriores, estaba dedicado a ampliación de teoría respecto a las asignaturas de Optometría I y Optometría II.

Respecto a la segunda pregunta que se centraba el valoración de la capacidad de diagnóstico en patologías optométricas, los alumnos aumentaron significativamente los resultados de aprendizaje ellos mismos marcaron cómo habían podido aumentar su capacidad para diagnosticar y definir qué tipo de patología tenían los pacientes. En cuanto la última pregunta, se observó un aumento muy grande en el aprendizaje. Este realmente era el objetivo y 
la finalidad de la asignatura, mejorar la capacidad de los alumnos para elaborar un plan de tratamiento para pacientes y realizar un seguimiento. Además, a nivel individual y subjetivo los alumnos me trasladaron sus opiniones sobre el transcurso del ciclo de mejora. Finalizado el cuatrimestre quiero recoger estas opiniones de manera anónima para poder analizarlas y estudiarlas. Ellos mismos me trasladaron las ventajas de poder ver casos reales y aplicar la teoría ya explicada en cursos anteriores.

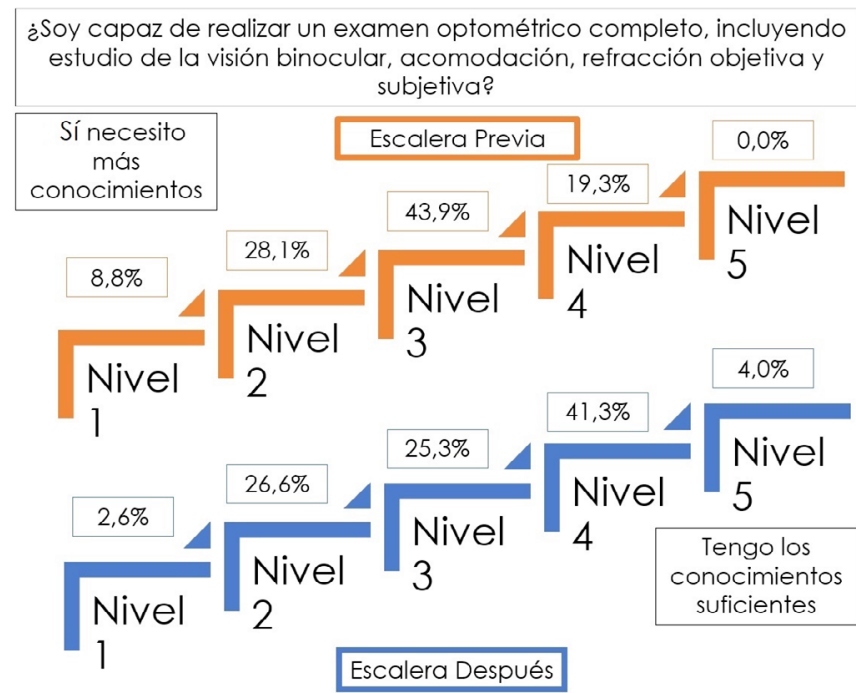

Tengo los conocimientos suficientes para diagnosticar a los pacientes de problemas optométricos

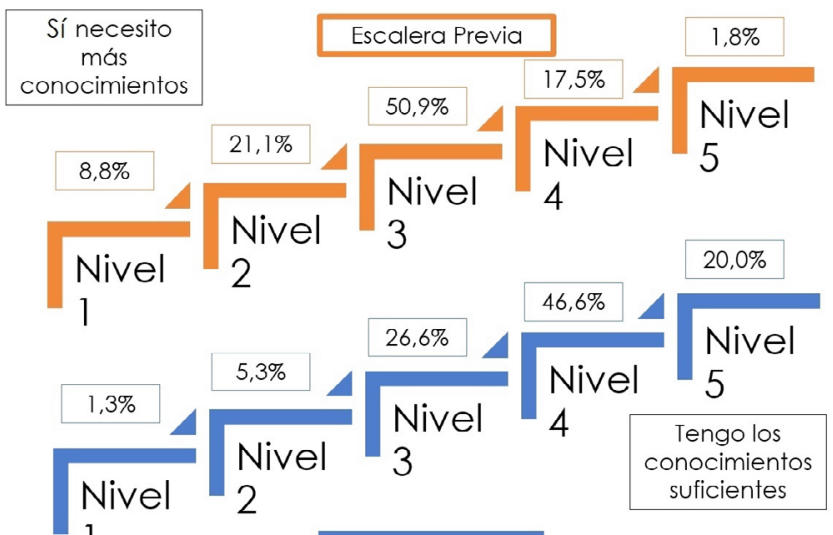

Escalera Después

Jornadas de Formación e Innovación Docente del Profesorado | № 1 (2018) Esta obra se distribuye con la licencia Creative Commons Reconocimiento-NoComercial-SinObraDerivada Internacional (CC BY-NC-ND 4.0.) 
Tengo los conocimientos suficientes para elaborar un tratamiento optométrico desde el punto de vista refractivo o de terapia visual

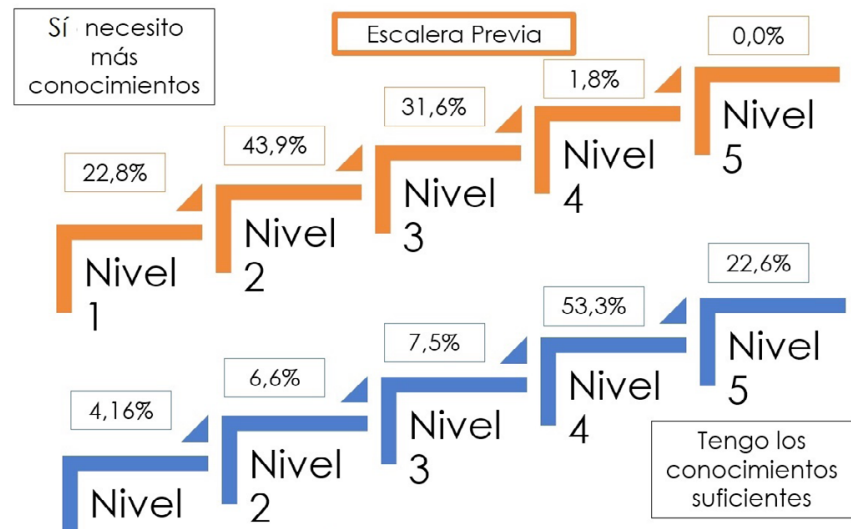

Escalera Después

Figura 5. Escaleras de aprendizaje para las 3 preguntas del cuestionario

\section{Evaluación del CMD}

Respecto a las cuestiones que mantendría para el siguiente CMD sería invertir el orden de la teoría y la práctica. Creo que ha resultado muy positivo que los alumnos aborden contenidos teóricos desde la resolución de casos clínicos. Como resultado, he visto como los contenidos teóricos han quedado relegados a un segundo, incluso tercer plano. Los contenidos teóricos fueron proporcionados mediante la plataforma, pero no los he explicado explícitamente durante la clase. Ningún alumno se ha quejado y han comprendido que era contenido para examen. Ya que sin los conceptos teóricos no se podían resolver los casos y construir las tesis oportunas sobre los mismos.

Otra de las cuestiones que mantendría es subir los casos clínicos al menos dos días antes de las clases. Los alumnos suelen esperar el contenido y se lo preparan con algo de antelación. Así me lo han hecho saber en comunicaciones que hemos podido tener entre nosotros. Subir el contenido con antelación pensé que supondría descenso 
en la asistencia. Para mi sorpresa, fue, al contrario. Por lo que será algo, que, dentro de mis posibilidades de tiempo, lo aplicaré en siguientes ciclos de mejora.

Respecto al segundo ciclo de mejora, lo mantendré igual, ya que se explicó en la comunicación de las Jornadas anteriores y sirve como base para luego abordar las prácticas con pacientes reales. Los alumnos me transmiten la inseguridad que tienen cuando realizan las pruebas a pacientes que no conocen o que son mayores que ellos. Por lo tanto, estos seminarios prácticos que se dan al inicio de curso les sirven a ellos para acercarse a la práctica con pacientes reales a través de repaso de los contenidos procedimentales con sus compañeros (Austin, 2003). Algo que también quiero mantener para otros cursos, es que los compañeros con los que se ponen para realizar las prácticas de seminarios sean los compañeros que no son los habituales.

Respecto a los cambios que se van a introducir, el primero y principal, será quitar la sesión de teoría al final de los 5 primeros casos clínicos, ya que me he dado cuenta de que es repetitivo y los alumnos no prestan atención. Realmente mientras se exponen los casos clínicos yo voy introduciendo pequeños aspectos teóricos, pero que no los llamo teoría como tal y me doy cuenta de que luego no es necesario una clase específica para tal finalidad.

Otra de las mejoras es reducir los grupos de seminarios prácticos para que la docencia sea más efectiva.

Entre los aspectos de la práctica que van a incorporarse la docencia habitual se encuentran; realizar las clases basada en casos clínicos, favorecer la participación de los alumnos, dar las explicaciones mientras me voy moviendo por la clase y subo las gradas y paseo entre los alumnos, sin mantenerme estático y en posición de poder. También considero positivo evaluar cada clase cuando termina con un juego de gamificación. Me he dado cuenta 
de que cuando se evalúa inmediatamente después y se obtiene un resultado también inmediato los alumnos se encuentran más satisfechos con lo que hacen y las sensaciones obtenidas son más positivas.

Los principios didácticos que han guiado este ciclo de mejora han sido: nadie se educa o aprende por otra persona, de ahí que las actividades deban ser realizadas por el propio alumno, con nuestro seguimiento, pero de una manera autónoma; es imprescindible fomentar la creatividad, el alumno debe ir construyendo sus propias respuestas; el proyecto de la asignatura debe ir anticipándose a la realidad del paciente que van a tener en las prácticas, es decir, los contenidos deben ser abordados de manera que se perciba su funcionalidad para la profesión.

Por último, considero de aplicación en este ciclo de mejora como principio fundamental, el de socialización. El ser humano tiene que relacionarse con otras personas y la educación debe responder a las características y necesidades de la sociedad. Por lo que, en este caso, el alumno necesita socializarse con su medio (de aplicación con los pacientes reales que vamos a examinar).

\section{Conclusiones}

La metodología y el modelo metodológico personal que yo he usado puede favorecer enormemente la dinamización de la clase y el aprovechamiento de esta, de modo que los mismos alumnos se dan cuenta de que la clase fluye de manera dinámica y con aprendizaje continuo.

Desde mi punto de vista, esta metodología basa en aprendizaje por casos clínicos favorece el acercamiento al mundo profesional inminente de los alumnos. Esto supone un acercamiento y motivación del alumnado hacia la docencia. 


\section{Bibliografía}

Alderman, I. M. (2014). Teaching for Quality Learning at University. By John B. Biggs and Catherine Tang. New York, N.Y.: McGraw-Hill/Society for Research into Higher Education/Open University Press, 2011. xxii + 389 pages. ISBN 978-0-33-524275-7. \$41.00. Teaching Theology \& Religion, 17(1), 91-92. https://doi.org/10.1111/teth.12173

Ashwin, P. (2015). Reflective teaching in higher education. (C. P. Corporation, Ed.) (Continuum). Sidney: Corporation, Continuum Publishing.

Austin, A. E. (2003). Creating a Bridge to the Future: Preparing New Faculty to Face Changing Expectations in a Shifting Context The Review of Higher Education, 26(2), 119-144. https://doi.org/10.1353/rhe.2002.0031

Barnett, R., \& Society for Research into Higher Education. (2005). Reshaping the university : new relationships between research, scholarship and teaching. Society for Research into Higher Education/Open University Press.

Biggs, J. B. (John B. (2003). Teaching for quality learning at university: what the student does. Society for Research into Higher Education.

Elton, L. (2001). Research and Teaching: Conditions for a positive link. Teaching in Higher Education, 6(1), 43-56. https://doi.org/10.1080/13562510020029590

Elton, L. (2006). The nature of effective or exemplary teaching in an environment that emphasizes strong research and teaching links. New Directions for Teaching and Learning, 2006(107), 33-41. https://doi.org/10.1002/ tl. 243

Jackson, W. K., \& Simpson, R. D. (1994). Mentoring new faculty for teaching and research. New Directions for Teaching and Learning, 1994(57), 65-72. https://doi.org/10.1002/ tl.37219945709

Jaques, D. (2000). Learning in groups : a handbook for improving group working. Kogan Page.

Mizoguchi, R., Dillenbourg, P., \& Zhu, Z. (2006). Learning by effective utilization of technologies: facilitating intercultural understanding. IOS Press.

Musthafa, M. N. M. A., \& Sajila, K. M. (2014). Reconsidering the Teaching-Research Nexus in Higher Education. Higher Education for the Future, 1(2), 123-138. https://doi. org/10.1177/2347631114539871

Paroutis, S., Angwin, D., \& Heracleous, L. T. (n.d.). Practicing strategy: text \&amp; cases. 